\title{
Teoria da mente e habilidades sociais: estudo com crianças pré-escolares
}

\author{
Sara Del Prete Panciera \\ Ariane Caserta Zeller \\ Universidade Federal de São Paulo, SP, Brasil
}

\begin{abstract}
Resumo
A forma como a criança se relaciona socialmente está ligada à maneira como ela compreende o mundo social e, nesse sentido, busca-se conhecer como as mudanças que ocorrem na compreensão social da criança impactam seu comportamento na relação com outras pessoas. Este estudo teve o objetivo de verificar a existência de correlação entre o desenvolvimento de habilidades sociais e o da teoria da mente em crianças pré-escolares. Participaram da pesquisa 30 crianças de 5 anos de idade e suas professoras. Para a avaliação da teoria da mente foi utilizada a Escala de Tarefas, e para a avaliação das habilidades socais foi usado o Questionário de Comportamentos Socialmente Adequados, na sua versão para professores. Os resultados não mostraram associação entre as variáveis, sugerindo que a teoria da mente pode estar ligada a comportamentos sociais sem se expressar ou estar diretamente relacionada a um conjunto de habilidades sociais positivas.
\end{abstract}

Palavras-chave: Teoria da mente; Habilidades sociais; Desenvolvimento sociocognitivo.

\section{Theory of mind and social skills: a study with young children}

\begin{abstract}
The way children relate socially is linked to the way they understand the social world. It is studied how the changes that occur in the child's social understanding impact their behavior with other people. This study aimed to verify the existence of a correlation between the development of social skills and the theory of mind in preschool children. Thirty five-year-old children and their teachers participated in the study. For the evaluation of the theory of mind the theory-of-mind scale was used, and for the evaluation of social skills the Socially Appropriate Behavior Questionnaire, in its version for teachers, was used. The results showed no association between the variables, suggesting that the theory of mind can be linked to social behaviors without being directly related to a set of positive social skills.
\end{abstract}

Keywords: Theory of mind; Social skills; Sociocognitive development.

\section{Teoría de la mente y habilidades sociales: estudio con niños pré-escolares Resumen}

La forma en que los niños se relacionan socialmente está relacionada con la forma en que entienden el mundo social social y, en ese sentido, se busca conocer cómo los cambios que ocurren en la comprensión social del niño impactan su comportamiento en la relación con otras personas. Este estudio tuvo como objetivo verificar la existencia de correlación entre el desarrollo de habilidades sociales y la teoría de la mente. Treinta niños de cinco años de edad participaron en el estudio. Para la evaluación de la teoría de la mente se utilizó la Escala de Tareas, y para la evaluación de las habilidades sociales el Cuestionario de Comportamientos Socialmente Adecuados, en su versión para profesores. Los resultados no mostraron asociación entre las variables, sugiriendo que la teoría de la mente puede estar ligada a comportamientos sociales sin expresarse o estar directamente relacionada con un conjunto de habilidades sociales positivas.

Palabras clave: Teoria de la mente; Habilidades sociales; Desarrollo sociocognitivo. 


\section{Introdução}

A forma como as pessoas se comportam e as maneiras pelas quais atribuem sentido aos comportamentos dos outros estão intrinsecamente conectados à sua compreensão do mundo social. A compreensão social é sustentada por habilidades sociocognitivas, entre elas a teoria da mente, que é à habilidade de atribuir estados mentais a si e ao outro (Wimmer \& Perner, 1983). Atribuir estados mentais como desejos, intenções e crenças às outras pessoas é uma prática cotidiana, e, mesmo que raramente as pessoas se deem conta disso, é uma habilidade que nos permite dar sentido ao mundo social.

No início da década de 80 foram criadas as primeiras tarefas experimentais para a avaliação da habilidade de atribuição da falsa crença, um dos aspectos da teoria da mente (Domingues, Valério, Panciera, \& Maluf, 2007; Wimmer \& Perner, 1983), o que permitiu a realização de pesquisas que mostraram que as crianças começam a manifestar a habilidade de atribuir estados mentais por volta dos 3 anos de idade (Martins, Barreto, \& Castiajo, 2014). Durante aproximadamente três décadas a falsa crença foi o aspecto mais explorado pelos estudos sobre teoria da mente. Atualmente, entretanto, o instrumento mais frequentemente utilizado nas pesquisas experimentais nesse campo tem sido a escala de tarefas de teoria da mente, elaborada por Wellman e Liu (2004). A escala se constituiu como um instrumento importante para avaliar a teoria da mente, capaz de evidenciar a multiplicidade de fatores envolvidos no curso de seu desenvolvimento, pois, além da falsa crença, permite também avaliar as habilidades de atribuição de desejos, crenças e crença/emoção (Hiller, Weber, \& Young, 2014; Kuntoro, Saraswati, Peterson, \& Slaughter, 2013; O'Reilly \& Peterson, 2014; Shahaeian, Nielsen, Peterson, \& Slaughter, 2014).

A elaboração dessa escala contribuiu para a compreensão de que a aquisição de uma teoria da mente depende de um extenso e progressivo conjunto de aquisições conceituais, além de apontar para o fato de que nenhuma tarefa, isoladamente, poderia dar subsídios suficientemente fortes para analisar uma progressão do desenvolvimento (Wellman \& Liu, 2004). A escala, portanto, representou um avanço importante para a instrumentalização dos pesquisadores da sociocognição.

No estudo sobre o desenvolvimento da teoria da mente, várias interfaces têm sido exploradas, sendo uma delas a relação entre compreensão social e comportamento social (Martins, Barreto, \& Castiajo, 2014; Repacholi \& Slaughter, 2004). A questão que se que investiga nesse sentido é como as mudanças que ocorrem na forma da criança compreender o mundo social impactam seu comportamento, especialmente no que diz respeito ao convívio com outras pessoas.

\section{Habilidades sociais}

Habilidades sociais são comportamentos sociais de diversas classes que compõem o repertório do indivíduo, ajudando-o a lidar com as mais diferentes demandas do convívio interpessoal. As habilidades sociais são aprendidas e dependem do contexto histórico-cultural e das dimensões pessoais em que os indivíduos estão inseridos (Del Prette, A. \& Del Prette, Z., 2001; Pizato, Marturano, \& Fontaine, 2014).

Del Prette, A. e Del Prette, Z. (2001) destacam as principais classes nas quais pode ser dividido o conjunto de habilidades sociais relevantes: habilidades de comunicação (fazer e responder perguntas; iniciar, manter e finalizar uma conversa; elogiar), habilidades de civilidade (agradecer; pedir por favor; apresentar-se), habilidades assertivas de enfrentamento (expressar opinião; fazer e recusar pedidos; lidar com críticas; lidar com opiniões diferentes; interagir com autoridades), habilidades empáticas e de expressão de sentimento positivo (ser simpático; expressar alegria), habilidades sociais profissionais (falar em grupo; coordenar grupos; lidar com lideranças), habilidades sociais educativas (de pais; professores).

Comportamentos socialmente habilidosos podem ser vistos como repertórios "desejados" ou socialmente adequados. Segundo Bolsoni-Silva (2003), eles são entendidos como facilitadores do desenvolvimento, pois permitem a ocorrência de "saltos" comportamentais, ou seja, mudanças que vão ocorrer ao longo do processo de desenvolvimento e que dependem da interação organismo-ambiente (Bolsoni-Silva \& Mariano, 2014; Bolsoni-Silva, Marturano, \& Manfrinato, 2005). Essas mudanças seriam importantes para a criança, porque permitiriam a ela entrar em contato com novos desafios de aprendizagem, levando ao seu desenvolvimento. Além disso, as habilidades sociais, por favorecerem uma boa interação entre as pessoas e aumentarem a chance de acesso aos reforçamentos sociais como atenção e elogios, promoveriam o desenvolvimento e podem evitar o que pode ser entendido, por pais ou professores, como problemas de comportamento.

Por outro lado, comportamentos socialmente pouco habilidosos seriam "indesejados" e dificultariam os "saltos" de desenvolvimento do indivíduo (BolsoniSilva et al., 2005). Eles podem se manifestar sob duas formas: internalizantes ou externalizantes (Del Prette, 2005). Comportamentos internalizantes são evidenciados por retraimento, disforia e ansiedade. 
Eles diminuem as possibilidades de interação do indivíduo com o ambiente e geralmente são mais vistos em meninas. Comportamentos externalizantes são caracterizados pela agressão, impulsividade, agitação e comportamentos desafiantes e antissociais. Neste caso, eles podem gerar conflitos e uma consequente ruptura com as interações sociais. São mais vistos em meninos e ganham destaque no ambiente escolar, pois influenciam na dinâmica da sala de aula.

\section{Teoria da mente e habilidades sociais}

É através da socialização que a criança tem acesso a novas informações sobre o ambiente e sobre as pessoas ao seu redor. À medida que a criança vai desenvolvendo uma "linguagem da mente", vai se tornando mais capaz de entender como o comportamento humano pode ser influenciado pelos diferentes estados mentais e, ao mesmo tempo, também vai sendo introduzida em uma comunidade de mentes (Nelson, 2007).

Considera-se a teoria da mente como uma habilidade indissociável de uma rede social e cultural, o que evidencia a relevância de se investigar como as diversas características dos contextos nos quais as crianças estabelecem suas relações sociais podem influenciar a aquisição desta habilidade (Freitas, O'Brien, Nelson, \& Marcovitch, 2012; GomezGaribello \& Talwar, 2015). A forma como as crianças se relacionam se desenvolverá em função da qualidade das características das relações de que ela participa. Pais e professores, portanto, devem estar atentos, por exemplo, para a criação de regras justas, favorecedoras do desenvolvimento de habilidades pró-sociais. Segundo Del Prette, A. e Del Prette, Z. (2001), as habilidades sociais educativas de pais e professores possuem grande peso na promoção do repertório da criança e do adolescente.

A forma como a criança se relaciona socialmente está fortemente ligada à maneira como ela compreende o mundo social. Neste sentido, alguns autores (Ronald, Happé, Hughes, \& Plomin, 2005) identificaram três tipos de comportamentos, que se configuram tanto em termos de seu caráter pró ou antissocial, quanto em função do quanto exigiriam de compreensão a respeito da mente do outro: (a) comportamentos pró-sociais que exigem uma teoria da mente (e.g. considerar os sentimentos de outras pessoas), (b) comportamentos antissociais que exigem uma teoria da mente (e.g. culpar os outros por alguma coisa) e (c) comportamentos pró e antissociais que não exigem uma teoria da mente (e.g. destruir as coisas dos outros, ser gentil com animais).

É importante notar que os estudos têm evidenciado que uma compreensão da mente do outro pode tanto ser preditora de comportamentos socialmente adequados quanto de atitudes socialmente repreensíveis (Freitas, O'Brien, Nelson, \& Marcovitch, 2012; Gomez-Garibello \& Talwar, 2015; Pavarini \& Souza, 2010). Uma teoria da mente mais sofisticada pode estar relacionada, por exemplo, a comportamentos agressivos, como o bullying. A desigualdade de poder própria do bullying, que implica em uma relação de dominação, tem sido associada a uma capacidade bem desenvolvida de compreender a mente do outro (Stellwagen \& Kerig, 2013; Sutton, 2004).

Dentro do quadro amplo de pesquisas sobre a relação entre compreensão social e comportamento social, o objetivo deste estudo foi o de investigar a existência de relação entre o desenvolvimento de uma teoria da mente e as habilidades sociais demonstradas por crianças de idade pré-escolar. Para isso, foram avaliadas as habilidades das crianças para atribuir estados mentais, bem como as habilidades sociais manifestadas por elas, a partir de relatos de suas professoras.

\section{Método}

\section{Participantes}

Participaram do estudo 30 crianças ( 15 meninas e 15 meninos), de 5;1 a 5;11 anos de idade, frequentadoras de uma escola municipal de Educação Infantil (EMEI). A escola se localiza na periferia da cidade de Santos (SP) e atende, em sua maioria, filhos de trabalhadores do porto. Participaram da coleta de dados, também, as professoras das crianças.

Todos os procedimentos éticos foram observados e a pesquisa foi realizada mediante aprovação do Comitê de Ética em Pesquisa da Universidade Federal de São Paulo (CEP 1780/10). O estudo também foi aprovado pelo Comitê de Ética da Secretaria de Educação do Município de Santos (SEDUC) e realizado por meio da apresentação e assinatura de um termo de consentimento livre e esclarecido pelos responsáveis pelas crianças, bem como pelas professoras que participaram da pesquisa. As crianças foram convidadas a participar do estudo e esclarecidas, em linguagem apropriada, do seu direito de desistir da participação a qualquer momento.

\section{Instrumentos e procedimentos}

Para a avaliação das habilidades sociais em crianças pré-escolares, Bolsoni-Silva (2003) elaborou o Questionário de Comportamentos Socialmente Adequados, que possui uma versão para pais (QCSAPais) e uma versão para professores (QCSA-Pr). O questionário avalia as habilidades sociais manifes- 
tadas por crianças de 5 a 7 anos, não se ocupando especificamente de alunos que apresentem dificuldades em relação às habilidades sociais. Os questionários são respondidos a partir do que é avaliado pelos pais ou pelos professores em relação aos comportamentos de cada criança.

Neste estudo, os dados sobre as habilidades sociais das crianças foram obtidos pelo Questionário de Comportamentos Socialmente Adequados professores (QCSA-Pr). O questionário é composto por 24 questões que representam descrições de comportamentos possíveis de serem apresentados por criança (por exemplo, "faz pedidos?", "presta ajuda ao professor e colegas?" "faz amigos?"). As respostas dos professores, referentes aos comportamentos das crianças, podiam ser: não se aplica, se aplica pouco ou certamente se aplica.

As professoras responderam ao questionário na própria escola, individualmente, em local e horário escolhidos por elas.

Para a avaliação da teoria da mente, as crianças realizaram as sete tarefas da escala de teoria da mente criada por Wellman e Liu (2004), como adaptada por Panciera (2007) para ser utilizada com crianças brasileiras:

1. Desejos diferentes: A criança deve apreciar a ação do outro, quando ambos - a própria criança e o outro - apresentarem desejos diferentes sobre os mesmo objetos.

2. Crenças diferentes: A criança deve apreciar a ação do outro quando duas pessoas, a própria criança e o outro, têm crenças diferentes sobre os mesmos objetos e quando a criança não sabe qual crença é verdadeira ou falsa.

3. Acesso ao conhecimento: A criança vê o que há em uma caixa e deve avaliar se a outra pessoa que não teve acesso ao conteúdo da caixa conhece seu conteúdo.

4. Falsa crença (conteúdo): A criança deve avaliar a crença falsa de outra pessoa sobre o que há em um recipiente identificável (como uma caixa de band-aid), quando a criança sabe que o conteúdo não é o esperado para aquele recipiente particular.

5. Falsa crença (explícita): A criança deve apreciar onde alguém vai procurar determinado objeto, sendo que o personagem tem uma crença equivocada a respeito do local.

6. Crença-emoção: A criança deve avaliar como uma pessoa se sentirá em uma situação de falsa crença.

7. Emoção real-aparente: A criança deve avaliar se uma pessoa pode se sentir de uma determinada forma, mas aparentar uma emoção diferente.
As tarefas foram realizadas com as crianças individualmente, em uma única sessão de aproximadamente 15 minutos.

\section{Procedimentos de análise}

No instrumento Questionário de Comportamentos Socialmente Adequados - professores (QCSA-Pr), as respostas dos professores sobre os comportamentos das crianças foram transformadas em escores: a resposta não se aplica recebeu o escore zero; a resposta se aplica pouco recebeu o escore 1; e, a resposta certamente se aplica recebeu o escore 2. Assim, a pontuação das crianças no instrumento variou de zero (pontuação mínima) até 48 (pontuação máxima).

$\mathrm{Na}$ escala de teoria da mente, cada uma das sete tarefas da escala foi pontuada com zero (0) quando a criança não manifestava a habilidade de atribuir estados mentais, ou um (1), quando a criança manifestava essa habilidade. Dessa forma, os escores das crianças na escala variaram de zero (pontuação mínima) até 7 (pontuação máxima). Atentou-se também particularmente para o acerto das crianças especificamente na Tarefa 5 da escala, que corresponde à clássica tarefa de falsa crença encontrada na literatura.

\section{Resultados e Análise}

Foram obtidos dados referentes às habilidades sociais, pela utilização do Questionário de Comportamentos Socialmente Adequados - QCSA-Pr (0-48); e, à teoria da mente, pela utilização da Escala de tarefas de teoria da mente (0-7).

A Tabela 1 apresenta os dados descritivos do desempenho das 30 crianças no QCSA-Pr e na Escala de teoria da mente (Escala-ToM).

TABELA 1

Medidas descritivas do desempenho nos instrumentos de Habilidades Socias (QCSA-Pr) e na Escala de tarefas de Teoria da Mente (Escala-ToM)

\begin{tabular}{lcccc}
\hline & Média & $\begin{array}{c}\text { Desvio- } \\
\text { padrão }\end{array}$ & Mínimo & Máximo \\
\hline QCSA-Pr & 37,10 & 9,67 & 19 & 48 \\
Escala-ToM & 4,63 & 1,69 & 1 & 7 \\
\hline
\end{tabular}

Para verificar a associação entre os escores totais do QCSA-Pr e da Escala de teoria da mente utilizou-se o coeficiente de correlação linear de Pearson, que indicou não haver associação entre as variáveis $(0,276)$ $[-0,093 ; 0,579]$.

Considerando-se que a habilidade mais frequentemente investigada no desenvolvimento da teoria 
da mente é a de atribuição de falsa crença, foram analisadas as médias e desvios-padrão dos escores do QCSA-Pr para as crianças que erraram e para as que acertaram a Tarefa 5 (T5) da escala, a tarefa de falsa crença (explícita). Além de mais relacionada ao desenvolvimento da linguagem, a falsa crença de primeira ordem é também um indicador importante da compreensão social da criança.

A Tabela 2 apresenta os resultados no QCSA-Pr para as crianças que erraram e para as que acertaram a Tarefa 5 da escala.

TABELA 2

Medidas descritivas no instrumento de Habilidades Socias (QCSA-Pr), em função do desempenho na Tarefa 5 (T5) da Escala de teoria da mente (ToM)

\begin{tabular}{lcc}
\hline Resultados no & \multicolumn{2}{c}{ Desempenho na Tarefa 5 da escala de ToM } \\
\cline { 2 - 3 } QCSA-Pr & $\begin{array}{c}\text { Participantes que } \\
\text { erraram a T5 }\end{array}$ & $\begin{array}{c}\text { Participantes que } \\
\text { acertaram a T5 }\end{array}$ \\
\hline Média & 38,22 & 35,42 \\
Desvio-padrão & 9,91 & 9,45 \\
Mínimo & 19,00 & 19,00 \\
Máximo & 48,00 & 48,00 \\
\hline
\end{tabular}

Para o tratamento estatístico da relação entre o desempenho na Tarefa 5 e os escores no QCSA-Pr foi utilizado o teste $\mathrm{t}$ de Student para amostras não relacionadas. Obteve-se nível descritivo de 0,442, indicando que estas variáveis não apresentaram associação significativa, ou seja, os escores médios de habilidades sociais obtidos pelas crianças que erraram a Tarefa 5 não diferiram estatisticamente dos escores obtidos pelas crianças que acertaram esta tarefa.

Em face desses resultados, e considerando-se a literatura na área, aventa-se a hipótese de que a teoria da mente estaria relacionada a algumas habilidades sociais específicas e não ao conjunto total delas, como avaliado neste estudo. Alguns autores (Pavarini, Loureiro, \& Souza, 2011; Ronald, Happé, Hughes, \& Plomin, 2005) discutem que certas classes de habilidades sociais dependeriam mais de uma teoria da mente do que outras, ou seja, que a teoria da mente estaria ligada a classes de habilidades sociais mais dependentes da compreensão do mundo social (e.g. lidar com opiniões diferentes) que outras (e.g. pedir "por favor" ou apresentar-se).

Os resultados deste estudo sugerem que o desenvolvimento de uma teoria da mente nas crianças não estaria ligado de forma direta a todos os comportamentos pró-sociais. Colocado dentro do quadro maior dos comportamentos sociais, focalizar as habilidades sociais, entendidas como repertório desejável ou socialmente adequado de comportamentos, pode ser insuficiente para compreender a motivação dos comportamentos e o papel da teoria da mente nessa complexa relação. Nesta perspectiva, os resultados deste estudo, que não indicaram haver relação entre teoria da mente e habilidades sociais avaliadas pela professora das crianças, sinalizam que os comportamentos precisam ser vistos e analisados dentro do contexto em que são produzidos.

Pesquisas recentes têm, por exemplo, explorado relações entre a teoria da mente, a necessidade de se fazer inferências para identificar o estado emocional do outro e a empatia (Pavarini \& Souza, 2010). Por outro lado, Sutton (2004) aponta o bullying como um comportamento relacionado positivamente ao desenvolvimento de uma teoria da mente. Isso implica que a compreensão da criança de que o outro tem representações diferentes da sua própria não implica que ela irá se solidarizar com este outro. Também parece parcial e incorreto afirmar que o praticante do bullying seja socialmente incompetente, visto que um dos efeitos desse comportamento muitas vezes é provocar (e conseguir) a admiração de outras pessoas a sua volta. Assim, reconhecer a ligação entre teoria da mente e comportamentos sociais não implica que esses comportamentos estejam dentro de um repertório considerado desejável. Levar em conta a interação das crianças em diferentes contextos, portanto, amplia a discussão sobre a maneira como a relação entre teoria da mente e comportamentos sociais se dá.

Outro aspecto revelado pelos resultados desta pesquisa, é que se identificou que, aos 5 anos, todas as crianças participantes tinham, em alguma medida, desenvolvido habilidades sociais e de teoria da mente. Os resultados das crianças nas tarefas de teoria da mente mostram que, na faixa etária estudada, elas estão em processo de construção dessas habilidades sociocognitivas. Verificou-se, por exemplo, que os acertos das crianças nas tarefas da escala de teoria da mente variaram, nos escores, de 1 a 7 (Tabela 1). Considerar essa construção processual corrobora outros achados na literatura (Shahaeian, Peterson, Slaughter, \& Wellman, 2011), que argumentam em favor da importância da cultura na constituição do indivíduo e no seu desenvolvimento sociocognitivo. As experiências anteriores das crianças, bem como o contexto sociocultural em que estão inseridas são apontadas em muitos estudos (Deleau, 2012; Morgan, Meristo, Mann, Hjelmquistc, Suriand, \& Siegal, 2014; Panciera, 2007; Panciera \& Domingues, 2013; Rodrigues, Pelisson, Silveira, Ribeiro, \& Silva, 2015) como fatores importantes para o desenvolvimento de uma teoria da mente. 
Os resultados mostraram ainda que aos 5 anos de idade, as crianças estão construindo um repertório de habilidades sociais que também ocorre de forma processual, à medida que a criança vai experimentando diferentes situações no contexto de seu grupo social. Assim, comportamentos que não condizem com um escore alto no instrumento de habilidades sociais, como atitudes agressivas ou outros comportamentos externalizantes, podem ser encontrados em crianças com desenvolvimento típico na fase pré-escolar, sem que isso implique em qualquer dificuldade particular da criança (Bolsoni-Silva \& Mariano, 2014; BolsoniSilva, Marturano, \& Manfrinato, 2005).

\section{Conclusão}

Este estudo teve o objetivo de verificar a existência de relação entre o desenvolvimento de habilidades sociais e o da teoria da mente em crianças préescolares. Os dados encontrados indicaram não haver correlação entre as variáveis, como investigadas, o que, em nossa análise, sinaliza para a complexidade dos fatores envolvidos na trajetória do desenvolvimento. Esses resultados contribuem no sentido de sinalizar que a compreensão do mundo social pode não se expressar diretamente em habilidades sociais positivas (como comportamentos desejáveis, socialmente adequados e culturalmente referenciados) e que essa relação deve ser mais bem explorada, em futuras pesquisas, no quadro amplo da relação entre compreensão social e comportamento social.

É importante notar que para esta pesquisa foi utilizada uma forma particular de verificação das habilidades sociais, a saber, a avaliação das professoras das crianças da Educação Infantil. Outras formas de avaliação, como a feita pelos pais/responsáveis ou a observação das crianças em situações menos ou mais estruturadas, podem ser reveladoras de outros aspectos relevantes do desenvolvimento. Além disso, atentar para as diversas classes de habilidades sociais - diferente do que foi feito no presente estudo, em que elas foram consideradas de maneira global - pode também ajudar a avançar na investigação do desenvolvimento da compreensão do mundo social.

No estudo também se verificou que, aos 5 anos, todas as crianças estavam em alguma medida desenvolvendo as habilidades avaliadas, ou seja, em um período crítico de sua trajetória desenvolvimental. Considerando nosso contexto social e educativo, é importante lembrar que crianças com comportamentos socialmente pouco habilidosos, principalmente no que se refere aos comportamentos externalizantes, podem ser mal interpretadas por pais e educadores, justamente em um período de exercício e desenvolvimento dessas habilidades. Os adultos podem mudar seu comportamento junto à criança, no sentido de incluí-la menos nas conversas explicativas, nas conversas sobre emoções (Dunn, Brown, \& Beardsall, 1991), bem como diminuir o acesso dessa criança aos reforçamentos sociais (Bolsoni-Silva, Marturano, Pereira, \& Manfrinato, 2006), o que teria efeitos no processo de desenvolvimento tanto das habilidades sociais quanto da teoria da mente, e que, em determinados cenários, pode amplificar um contexto de vulnerabilidade.

Os resultados apontam, assim, para a importância de práticas, especialmente práticas educativas no contexto da Educação Infantil, que não "normalizem" as crianças pré-escolares, no sentido de considerar patológicos alguns comportamentos identificados como socialmente pouco habilidosos, dado que elas estão em processo de exercício e aprendizagem de habilidades sociais.

Investigar o desenvolvimento sociocognitivo buscando conhecer as relações entre teoria da mente e habilidades sociais nos informa sobre questões centrais nos comportamentos cotidianos, isso porque compreender estados mentais de outras pessoas, bem como conseguir manter comportamentos que favoreçam as relações nos contextos de que se participa e com as pessoas com quem se convive são recursos fundamentais que sustentam a vida social.

\section{Referências}

Bolsoni-Silva, A. T. (2003). Habilidades Sociais Educativas, variáveis contextuais e problemas de comportamento: comparando pais e mães de pré-escolares (Tese de Doutorado). Universidade de São Paulo, Ribeirão Preto, SP, Brasil.

Bolsoni-Silva, A. T. \& Mariano, M. L. (2014). Práticas educativas de professores e comportamentos infantis, na transição ao primeiro ano do Ensino Fundamental. Estudos e pesquisas em psicologia, 14(3), 814-833. https://doi.org/10.12957/ epp.2014.13912

Bolsoni-Silva, A. T., Marturano, E. M., \& Manfrinato, J. W. S. (2005). Mães avaliam comportamentos socialmente “desejados" e "indesejados" de pré-escolares. Psicologia em Estudo, 10(2), 245-252. https://doi.org/10.1590/S141373722005000200011 
Bolsoni-Silva A. T., Marturano, E. M., Pereira, V. A., \& Manfrinato, J. W. S. (2006). Habilidades sociais e problemas de comportamento de pré-escolares: comparando avaliações de mães e professoras. Psicologia: Reflexão e Crítica, 19(3), 460-469. https://doi.org/10.1590/S0102-79722006000300015

Deleau, M. (2012). Language and theory of mind: why pragmatics matter. European Journal of Developmental Psychology, 9(3), 295-312. https://doi.org/10.1080/17405629.2012.680303

Del Prette, Z. A. P. (2005). Psicologia das habilidades sociais na infância: teoria e prática. Avaliação Psicológica, 4(1), 91-93.

Del Prette, A. \& Del Prette, Z. A. P. (2001). Habilidades Sociais: Intervenções efetivas em grupo. São Paulo: Casa do Psicólogo.

Domingues, S. F. S., Valério, A., Panciera, S. D. P., \& Maluf, M. R. (2007). Tarefas de crença falsa na avaliação de atribuição de estados mentais de crença. In P. W. Schelini (Org.). Alguns domínios da avaliação psicológica. Campinas: Alínea.

Dunn, J., Brown, J., \& Beardsall, L. P (1991). Family Talk About Feeling States and Children's Later Understanding of Others' Emotions. Developmental Psychology, 27(3), 448-455. https://doi.org/10.1037/0012-1649.27.3.448

Freitas, L. B. L., O’Brien, M., Nelson, J. A., \& Marcovitch, S. (2012). A Compreensão da Gratidão e Teoria da Mente em Crianças de 5 anos. Psicologia: Reflexão e Crítica, 25(2), 330-338. https://doi.org/10.1590/S0102-79722012000200015

Gomez-Garibello, C. \& Talwar, V. (2015). Can you read my mind? Age as a moderator in the relationship between theory of mind and relational aggression. International Journal of Behavioral Development, 39(6), 552-559. https:// doi.org/10.1177/0165025415580805

Hiller, R. M., Weber, N., \& Young, R. L. (2014). The Validity and Scalability of the Theory-of-Mind Scale with Toddlers and Preschoolers. Psychological Assessment, 26(4), 1388-1393. https://doi.org/10.1037/a0038320

Kuntoro, I. A., Saraswati, L., Peterson, C., \& Slaughter, V. (2013). Micro-cultural influences on theory of mind develpments: a comparative study of middle-class and pemulung children in Jakarta, Indonesia. International Journal of Behavioral Development, 37(3), 266-273. https://doi.org/10.1177/0165025413478258

Martins, C., Barreto, A. L., \& Castiajo, P. (2014). Teoria da mente ao longo do desenvolvimento normativo: Da idade escolar até à idade adulta. Análise Psicológica, 1(23), 377-392.

Morgan, G., Meristo, M., Mann, W., Hjelmquistc, E., Suriand, L., \& Siegal, M. (2014). Mental state language and quality of conversational experience in deaf and hearing children. Cognitive Development, 29, 41-49. https://oi. org/10.1016/j.cogdev.2013.10.002

Nelson, K. (2007). Young minds in social worlds. UK: Harvard University Press. https://doi.org/10.4159/9780674041400

O'Reilly, J. \& Peterson, C. C. (2014). Scaling theory of mind developments in indigenous- and anglo-australian toddlers and older children. Journal of Cross-Cultural Psychology, 45(9), 1489-1501. https://doi.org/10.1177/0022022114542285

Panciera, S. D. P. (2007). Linguagem e desenvolvimento da teoria da mente: um estudo com crianças de 3 a 5 anos (Tese de Doutorado). Universidade de São Paulo, São Paulo.

Panciera, S. D. P. \& Domingues, S. F. S. (2013). Pragmática da linguagem e representação de crença. In A. Roazzi \& T. M. Sperb (Eds.). O desenvolvimento de competências sociocognitivas: novas perspectivas. São Paulo: Vetor.

Pavarini, G. \& Souza, D. H. (2010). Teoria da mente, empatia e motivação pró-social em crianças pré-escolares. Psicologia em Estudo, 15(3), 613-622. https://doi.org/10.1590/S1413-73722010000300019

Pavarini, G., Loureiro, C. P., \& Souza, D. H. (2011). Compreensão de emoções, aceitação social e avaliação de atributos comportamentais em crianças escolares. Psicologia: Reflexão e Crítica, 24(1), 135-143. https://doi.org/10.1590/ S0102-79722011000100016

Pizato, E. C. G.,. Marturano, E. M., \& Fontaine, A. M. G. V. (2014). Trajetórias de habilidades sociais e problemas de comportamento no ensino fundamental: influência da educação infantil. Psicologia: Reflexão e Crítica, 27(1), 189. https://doi.org/10.1590/S0102-79722014000100021

Repacholi, B. \& Slaughter, V. (2004). Individual Differences in Theory of Mind. New York: Psychology Press. https:// doi.org/10.4324/9780203488508

Rodrigues, M. C., Pelisson, M. C. C., Silveira, F. F., Ribeiro, N. .N., \& Silva, R. L. M. (2015). Evaluation of theory of mind: A study with students from public and private schools. Estudos de Psicologia, 32(2), 213-220. https://doi. org/10.1590/0103-166X2015000200006

Ronald, A., Happé, F., Hughes, C., \& Plomin, R. (2005). Nice and nasty theory of mind in preschool children: Nature and nurture. Social development, 14(4), 664-684. https://doi.org/10.1111/j.1467-9507.2005.00323.x

Shahaeian, A., Peterson, C. C., Slaughter, V., \& Wellman, H. M. (2011). Culture and the sequence of steps in theory of mind development. Developmental Psychology, 47(5), 1239-1247. https://doi.org/10.1037/a0023899

Shahaeian, A., Nielsen, M., Peterson, C. C., \& Slaughter, V. (2014). Iranian Mothers' Disciplinary Strategies and Theory of Mind in Children: A Focus on Belief Understanding. Journal of Cross-Cultural Psychology, 45(7), 1110-1123. https://doi.org/10.1177/0022022114534772

Stellwagen, K.. K. \& Kerig, P. K. (2013). Ringleader Bullying: Association with Psychopathic Narcissism and Theory of Mind Among Child Psychiatric Inpatients. Child Psychiatry Hum Dev, 44, 612-620. https://doi.org/10.1007/s10578012-0355-5 
Sutton, J. (2004). Tom goes to school: Social cognition and social values in bullying. In B. Repacholi \& V. Slaughter (Eds.). Individual Differences in Theory of mind. New York: Psychology Press. https://doi.org/10.4324/9780203488508

Wellman, H. M. \& Liu, D. (2004). Scaling Theory of Mind Tasks. Child Development, 75(2), 523-541. https://doi. org/10.1111/j.1467-8624.2004.00691.x

Wimmer, H. \& Perner, J. (1983). Beliefs about beliefs: representation and constraining function of wrong beliefs in young children's understanding of deception. Cognition, 13, 103-128. https://doi.org/10.1016/0010-0277(83)90004-5

Dados dos autores:

Sara Del Prete Panciera - Doutora, Universidade Federal de São Paulo.

Ariane Caserta Zeller - Psicóloga, Universidade Federal de São Paulo.

Endereço para correspondência:

Sara Del Prete Panciera

Unifesp - Departamento de Saúde, Educação e Sociedade

Rua Silva Jardim, 136 - Vila Mathias

11015-020, Santos, SP, Brasil

<sara.panciera@unifesp.br>

Recebido em: 26.06.2017

Aceito em: 04.11.2017 